\title{
Simulation of a NE213 Gamma Response Function using an Improved Multi-parameter Minimization Procedure
}

\author{
O. Litaize* \\ CEA Cadarache, DEN/DER/SPRC/LEPh Bat 230, 13108 Saint Paul lez Durance, France
}

Do not use this line: Received date here; revised date here; accepted date here

\begin{abstract}
The Gamma response functions of a $15 \mathrm{~cm}$ diameter $\mathrm{x} 6 \mathrm{~cm}$ thick NE213 detector were recently measured with standard ${ }^{22} \mathrm{Na}$, ${ }^{60} \mathrm{Co}$ and ${ }^{241} \mathrm{Am} \gamma$ sources in order to calibrate the scintillation counter and were simulated in the present work with the MCNPX2.5e Monte-Carlo code. The MINUIT2 package, developed at C.E.R.N., was used to minimize the $\chi^{2}$ between measured and calculated pulse height distributions in order to derive the experimental resolution parameters. A $C++$ code was written to simulate various resolution broadening and background shapes. The results are in good agreement with the experimental response function except in the low energy range (below $100 \mathrm{keV}$ ) which could be improved by a better description of the detector environment.
\end{abstract}

Keywords: NE213; Gamma Response Function; MINUIT2; Monte-Carlo; MCNPX PACS: 29.40.Mc; 32.80-t; 02.70.Lq

\section{Introduction}

The reliable simulation of an experiment often requires a precise knowledge of the detector resolution. This is particularly true in the case of the detection of prompt neutrons and prompt gammas emitted from excited fission fragments, as detected by a NE213 liquid organic scintillator. Its resolution function can be assessed through a robust iterative procedure by applying a proper broadening function on the raw Compton electron distribution, as accurately reproduced by Monte-Carlo calculations.

While other calculations were performed to simulate the neutron detector intrinsic efficiency (which requires the resolution parameters as input data) and the time-of-flight spectra for instance, the present paper focuses solely on the analysis of the gamma spectra.

\footnotetext{
*Corresponding author. Tel.:+33-4-4225-7257; fax:+33-4-4225-7009; e-mail: olivier.litaize@cea.fr
} 


\section{Experimental set-up}

The purpose of the experiment was the study of the fission mechanism through the measurement of fission fragments characteristics in coincidence with promptly emitted neutrons and gamma [1]. The measurements were performed at the Institute for Reference Materials and Measurements at Geel, in Belgium. A ${ }^{252} \mathrm{Cf}(\mathrm{sf})$ source was mounted on the cathode centre of a double Frisch-grid ionization chamber, used as a fission fragment detector. A $15 \mathrm{~cm}$ diameter x 6cm thick NE213 scintillator coupled to a XP2041 photo-multiplier was used to detect the neutrons and $\gamma$-rays produced by the fragments. The photo-multiplier anode and ionization chamber cathode signals were summed and sent to a wave form digitizer from FAST ComTec (200 Msamples/s) and stored on hard disk for off-line analysis.

For calibration purpose, through Compton edges, different $\gamma$-sources were mounted on the centre of the entrance window of the scintillator. In order to relate the channel number (corresponding to the integrated charge of the photo-multiplier pulses) to light outputs, the pulse height distributions from standard sources of ${ }^{22} \mathrm{Na},{ }^{60} \mathrm{Co}$ and ${ }^{241} \mathrm{Am}$ were measured. The two former spectra (see Fig. 1) were analyzed. The $\gamma$ energies are $1.275 \mathrm{MeV}, 0.511 \mathrm{MeV}$ for ${ }^{22} \mathrm{Na}$ and $1.333 \mathrm{MeV}, 1.173 \mathrm{MeV}$ for ${ }^{60} \mathrm{Co}$. The theoretical position of the Compton edges is given by the well known relation: $\mathrm{E}=2 \mathrm{E}_{\gamma}{ }^{2} /\left(\mathrm{m}_{\mathrm{e}} \mathrm{c}^{2}+2 \mathrm{E}_{\gamma}\right)$. The channel numbers are linearly related to the deposited electron energy through calibration points corresponding to $80 \%$ of the maximum of the Compton edges.

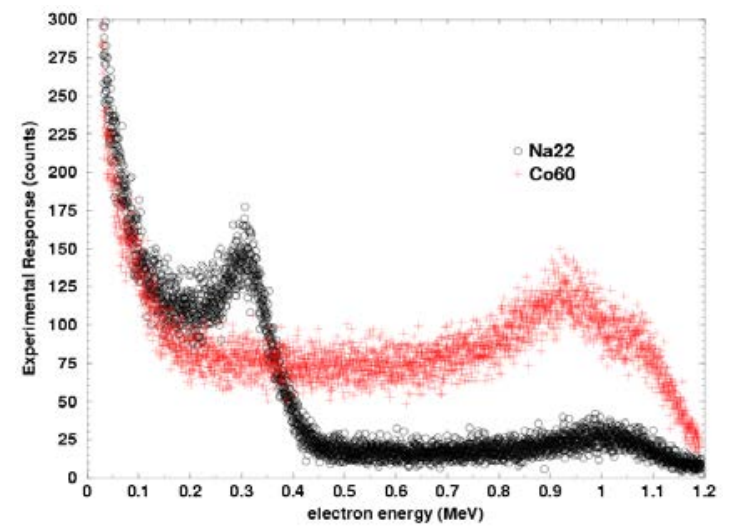

Fig. 1. Experimental Pulse Height Distributions.

\section{Simulation of the response function by MCNPX}

Because of additional requirements (e.g., neutron detection efficiency based on a proton pulse height light tally) for the complete simulation of this experiment, the MCNPX Monte Carlo transport code [2] was preferred to the well known MCNP code. Fig. 2 shows a transversal plot of the experimental setup simulated by MCNPX. The scintillator coupled to the photo-multiplier is located inside a conical shielding of paraffin. A collimator is inserted in the shielding. The $\gamma$ calibration sources were placed in front of the detector and replaced inside the shielding.

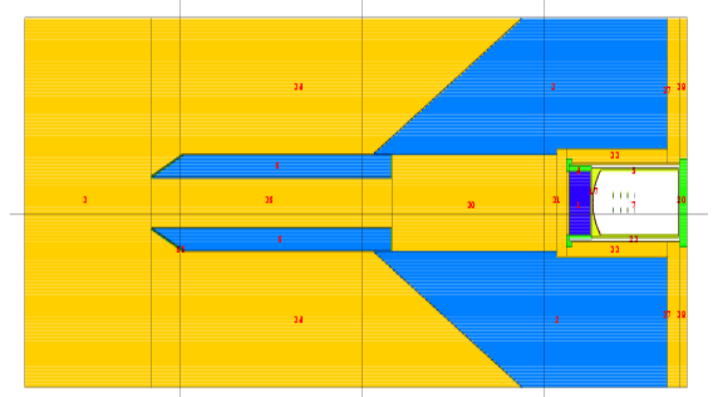

Fig. 2. Experimental set-up simulated by MCNPX2.5e.

The F8 pulse height tally modified by the PHL option was used to obtain the electron pulse height spectrum in the detector active cell. The statistical uncertainty is about $1 \%$ at $1 \mathrm{MeV}$ and $0.5 \%$ at $300 \mathrm{keV}$. The environment of the detector (shielding, collimator, aluminum housing...) was taken into account in the simulation and impacts the low energy range of the spectrum $(\mathrm{E}<100 \mathrm{keV})$. MCNPX has the capability to simulate a Gaussian broadened $\gamma$ spectrum but the 3-parameter Full Width at Half Maximum has to be known a priori. A possible way to find some parameters like those is based on an iterative procedure which takes as input data the experimental spectrum and a mathematical model involving the convolution of the calculated raw spectrum and a smearing function. The goal is simply to minimize the sum of the squares of the deviations between measured values and predictions of the model by adjusting the parameters. This can be performed through the MINUIT2 package [3]. 


\section{The MINUIT2 minimization package for spectrum broadening}

The MINUIT2 package is an open source library and can be used inside a home made $\mathrm{C}++$ code (a pure abstract base class FCNBase exists as interface).

Several minimization algorithms are available as part of MINUIT2. The most versatile for any kind of functions is MIGRAD which is a variable-metric method with inexact line search [4], a stable metric updating scheme and checks for positive definiteness.

The user must provide the so-called FCN multiparameter function in order to minimize the $\chi^{2}$ variable between the measured and calculated pulse height distributions, respectively $N^{\exp }\left(H, E_{\gamma}\right)$ and $N^{\text {calc }}\left(H, E_{\gamma}\right)$ where $H$ is the pulse height and $E_{\gamma}$ is the initial gamma energy. The pulse height scale is related to the scale of light outputs by the calibration procedure. The light output $L$ is related to the deposited electron energy $E$ by $L=E-E_{0}$ for energies $E \geq 40 \mathrm{keV}$, where $E_{0}$ is an energy offset equal to 5 $\mathrm{keV}$ which takes into account the non linearity due to quenching effects in liquid organic scintillators [5].

First of all, the experimental distribution is rebinned and mapped on the calculation grid (typically from 10000 to 200 mesh points). The raw calculated distribution $R\left(L, E_{\gamma}\right)$ is obtained from the $F 8$ pulse height light tally available in MCNPX. It is the probability that a $\gamma$ with an energy $E_{\gamma}$ deposits an energy $E$ corresponding to a light output $L$.

This raw calculated distribution is broadened by a Gaussian function $G$ with five parameters which characterizes the conversion process from light output to pulse height. In addition to that, a slight energy dependent correction is applied at low energy to the tail of the signal because this part of the signal is strongly affected by scattered gamma coming from the environment which can not be perfectly accounted for in the simulation.

Finally, the smeared calculated spectrum is a convolution product of these functions and can be compared to the experimental result:

$$
N^{\text {calc }}\left(H, E_{\gamma}\right)=\int_{0}^{\infty} R\left(L, E_{\gamma}\right) \eta L^{\zeta} G(H, L \mid \alpha, \beta, \gamma, \delta, \varepsilon) d L .
$$

In our case, the functional involves seven parameters that can be freely adjusted within certain constraints (e.g. with single/double sided limits, fixed or constant).

$\eta$ is a level adjustment parameter that can be initialized by comparing the 'plateau' of the raw calculated and experimental spectra (e.g., from 0.5 to $0.8 \mathrm{MeV}$ for ${ }^{22} \mathrm{Na}$ and from 0.3 to $0.7 \mathrm{MeV}$ for ${ }^{60} \mathrm{Co}$ ).

$\zeta$ allows to slightly correct the tail of the signal at low energy due to gamma backscattering from surrounding materials.

$\delta$ and $\varepsilon$ characterizes the mean of the Gaussian function $\langle H\rangle=\varepsilon L+\delta$ (calibration).

$\alpha, \beta$ and $\gamma$ are three parameters describing the Full Width at Half Maximum (FWHM) of the Gaussian related to the resolution by:

$$
R=\frac{F W H M}{L}=\sqrt{\alpha^{2}+\frac{\beta^{2}}{L}+\frac{\gamma^{2}}{L^{2}}},
$$

where the right hand side of the equation is the well known formula from references [5] and [6]. Other parameterizations have been tested and the procedure has been validated as follows.

As stated earlier, a Gaussian broadened spectrum can be obtained with MCNPX in a single run if the parameters of the FWHM are known (GEB option). In this way, two simulations were performed with and without broadening. The raw spectrum was convoluted with a Gaussian taking into account the same FWHM form than in the code $\left(a+b \sqrt{ }\left(E+c E^{2}\right)\right)$. Convergence was quickly achieved and the $a, b$ and $c$ values have been exactly reproduced.

In our case, the best agreement between experiment and calculation is reached with a constant resolution as a function of the deposited energy: $R=12 \%$. Numerous tests have been performed: adjusting the spectrum on a restricted part of the energy range, forcing the code to fix $\beta$ and $\gamma$ parameters at small values (non zero). In all cases, the minimum of the $\chi^{2}$ function has been obtained with $\beta, \gamma \rightarrow 10^{-6}$ and consequently with $R$ almost constant. Note that, although $R$ is expected to decrease with increasing energy (equation (2)), a constant $R$ value is an available option in the NEFF4 code [7] dedicated to the neutron detection efficiency calculation. Remind that this resolution is an input parameter for the calculation of the neutron efficiency of a NE213 counter. Two reasons may explain this situation. First, the eternal sources of 
annihilation quanta due to pair production in the surrounding materials can not be properly determined as mentioned above. This contribution affects the tail of the spectrum in the low energy range. In the case of the ${ }^{22} \mathrm{Na}$ source, the relative intensity of the annihilation quanta is not known and the Compton edge around $340 \mathrm{keV}$ is therefore slightly biased. The second reason is the existence of saturated pulses observed during the treatment of experimental data for high pulse heights around $1.2 \mathrm{MeV}$. Consequently for high and low energies some discrepancies may occur and affect the determination of the resolution parameters.

Despite these considerations the results are very satisfactory. The measured and calculated Compton electron pulse height spectra produced by $\gamma$-rays of the ${ }^{22} \mathrm{Na}$ and ${ }^{60} \mathrm{Co}$ sources are shown in Fig. 3 and Fig. 4 respectively (the number of experimental points has been decreased for clarity).

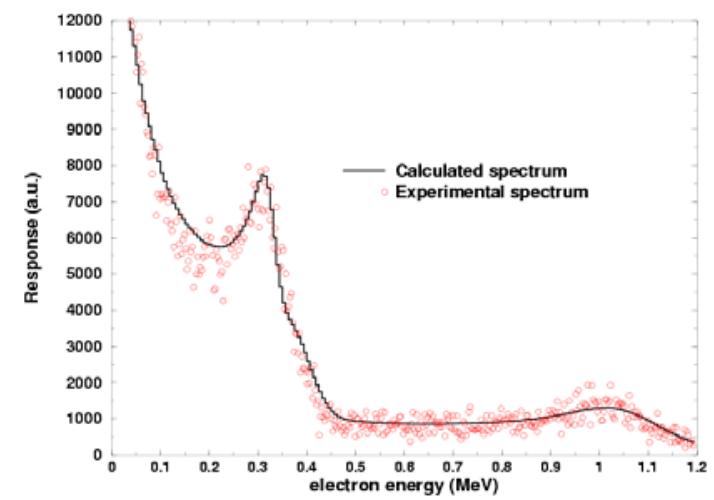

Fig. 3. Measured and calculated pulse height spectrum for Na22.

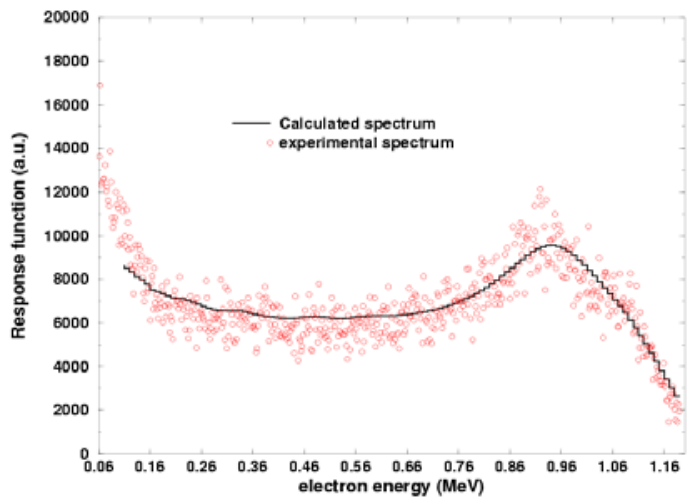

Fig. 4. Measured and calculated pulse height spectrum for Co60.
The parameters of the resolution obtained with the ${ }^{22} \mathrm{Na}$ source are very close to those obtained with the ${ }^{60} \mathrm{Co}$ source and then could be fixed in order to determine the other parameters more accurately.

\section{Summary}

Experimental pulse height distributions can be reproduced with the robust and highly flexible MINUIT2 package in association with accurate Monte Carlo simulations.

Even at low energy, a better agreement could be obtained with a refined representation of the source holder and others quantities which generate scattered photons impinging the detector. The neutron efficiency has also been calculated with the experimental resolution obtained in this work and is in good agreement with the measured efficiency.

In a near future, it could be advantageous to perform the complete analysis inside the ROOT system developed at C.E.R.N. It is an object-oriented framework used world wide for data analysis which includes the MINUIT2 package, among others.

\section{Acknowledgments}

The author wishes to thank $\mathrm{N}$. Varapai for providing her experimental results and is grateful to C. Suteau and C. de Saint-Jean for their help in the use of the STL $C++$ Standard Library.

\section{References}

[1] N. Varapai et al., Proc. of the $3^{\text {rd }}$ Int. Workshop on Nuclear Fission and Fission Product Spectroscopy, 369-372, Cadarache, France. 2006.

[2] J.S. Hendricks et al., LA-UR-04-0569, Los Alamos National Laboratory, Los Alamos, USA. February 2004.

[3] F. James and M. Winkler, http://www.cern.ch/minuit. C.E.R.N. May 2004.

[4] R. Fletcher, Computer Journal (1970), vol. 13, 317-322.

[5] G. Dietze, IEEE-Trans. NS-26 (1979), 398.

[6] G. Dietze, H. Klein, NIM 193 (1982), 549-556.

[7] G. Dietze, H. Klein, PTB-ND-22 (1982). 